\title{
HN1 Promotes Proliferation, Metastasis and Attenuates the Chemosensitivity of HCC Cells to Oxaliplatin by Inhibiting Degradation of HMGB1 Through Interacting With TRIM28
}

\section{Ruhua Wang}

Xi'an Jiaotong University Medical College First Affiliated Hospital

\section{Yunong Fu}

Xi'an Jiaotong University Medical College First Affiliated Hospital

\section{Menglin Yao}

Xi'an Jiaotong University Medical College First Affiliated Hospital Department of Medical Oncology

Xiaomeng Cui

Xi'an Jiaotong University Medical College First Affiliated Hospital

\section{Yan Zhao}

Xi'an Jiaotong University Medical College First Affiliated Hospital

\section{Xinlan Lu}

Xi'an Jiaotong University Medical College First Affiliated Hospital

\section{Yarui Li}

First Medical College: First Affiliated Hospital of Dalian Medical University

Shuixiang He ( $\nabla$ dyyyjxk@mail.xjtu.edu.cn )

First Affiliated Hospital of Xi'an Jiaotong University

\section{Research}

Keywords: HN1, HMGB1, cell proliferation, metastasis, oxaliplatin sensitivity

Posted Date: August 3rd, 2021

DOl: https://doi.org/10.21203/rs.3.rs-754224/v1

License: (9) This work is licensed under a Creative Commons Attribution 4.0 International License. Read Full License 


\section{Abstract}

Background: The oxaliplatin-based chemotherapy has revealed an encouraging therapeutic efficacy for advanced hepatocellular carcinoma patients. However, the development of resistance limits its clinical utilization. In addition, the chemotherapy resistance in HCC is usually accompanied with other malignant phenotypes, such as cell proliferation and metastasis, which together result in poor prognosis of HCC patients. Therefore, efforts should be made to explore potential regulators which fuel multiple events of HCC progression.

Methods: The qRT-PCR, western blot, immunohistochemistry and immunofluorescence were performed to measure mRNA and protein expression. MTT assay, colony formation and Transwell assay were performed to evaluate cell proliferation and metastasis. Flow cytometry was performed to test cell apoptosis. Alkaline Comet assay was performed to measure DNA lesions. Transmission electron microscope analysis provided potent testimony of autophagy. The role of HN1 on the malignant phenotypes of hepatoma carcinoma was demonstrated in vitro and in vivo.

Results: The immunohistochemistry analysis of HCC patient tissues revealed that the expression of HN1 was higher in HCC tissues compared to adjacent tissues and was associated with worse prognosis. In vitro, HN1 knockdown inhibited proliferation and metastasis of HCC cells, whereas HN1 overexpression promoted their proliferation and metastasis. In addition, we found that HN1 knockdown sensitized HCC cells to oxaliplatin, which is companied with deteriorated DNA damage and increased cell apoptosis in oxaliplatin-treated HCC cells. In vivo, HN1 knockdown inhibited the tumor growth and metastasis, and promoted the anti-cancer efficiency of oxaliplatin. Mechanically, HN1 prevented HMGB1 from ubiquitination and degradation via autophagy-lysosome pathway, which is related to its interaction with TRIM28, and overexpression of HMGB1 can restore the malignant phenotypes of HN1 knockdown in HCC cells. Furthermore, we found that HN1 can regulate cellular autophagy via HMGB1, which is important to tumor-promoting effect of HN1.

Conclusions: In conclusion, we systemically revealed the multiple functions of HN1 in HCC progression and the underlying molecular mechanism, which indicated that HN1 could be a promising therapeutic target for HCC treatment.

\section{Background}

Hepatocellular carcinoma (HCC) is the most common malignant hepatic disease, ranked as the sixth in tumor incidence and the third in leading cancer-related mortality worldwide [1]. Delay diagnosis and limited therapy deteriorate poor prognosis of advanced HCC patients. In decades, several therapeutic strategies have been developed to against advanced unresectable HCC. The oxaliplatin-based chemotherapy has revealed encouraging therapeutic efficacy $[2,3]$. However, HCC usually variously responses to chemotherapeutic agents because of different sensitivity of cancer cells, which limits the utilization of oxaliplatin-based chemotherapy. Therefore, identification of sensitivity regulators in HCC 
cells is urgent to optimize current chemotherapeutic strategies. In addition, the low sensitivity of HCC cells is usually accompanied with aggressive and proliferative phenotypes which also contribute to the poor prognosis of advanced HCC patients [4]. Therefore, finding a therapeutically targetable central regulator which controls multiple process in HCC progression should be seriously considered.

Oxaliplatin is the third generation platinum drug which mainly targets DNA, generates DNA adducts, causes inter- and intra-strand crosslinking (ICLs) and ends up with DNA damage [5]. In this process, unwound, bended and destabilized DNA duplex structure which can recruit DNA repair-associated proteins and activate DNA damage repair pathway. Indeed, activity of DNA damage repair pathways are closely associated with chemosensitivity of HCC cells. Thus, DNA repair regulators have been extensively investigated to dissect mechanism of sensitivity modulation and resistance formation. Among these regulators, HMGB1, a highly abundant chromatin-associated non-histone protein, has received improving attention. HMGB1 was identified to regulate multiple DNA damage repair pathways and its expression was negatively correlated to the cellular sensitivity to DNA-damaging agents [6]. Specially, previous studies have demonstrated HMGB1 could promote processing of ICLs via multiple repair pathways [7-9]. Deficiency of HMGB1 revealed increased genomic instability, DNA damage and cell death $[8,10,11]$. In addition to regulating DNA damage repair, a series of studies found that HMGB1 could modulate drug sensitivity via regulating autophagy [12-14]. Besides, HMGB1 also played a crucial role in proliferation, metastasis in various types of human cancer [15-17]. Despite of deep investigation in biological functions of HMGB1, only a few studies were related to its own regulation mechanism. In macrophage, activation of CB2R promoted the degradation of intracellular HMGB1 via the autophagy-lysosome pathway [18]. In HCC cells, previous work has reported that HMGB1 was degraded via chaperone mediated autophagy pathway [19]. However so far, upstream regulator of HMGB1 protein remains unknown.

Hematological and neurological expressed (HN1), locating on chromosome 17q25.2, encodes a 16.5 kilodalton protein which is ubiquitously expressed and highly conserved [20]. Increasing evidences indicated that HN1 showed an elevated expression level and was associated with poor prognosis in many tumors [21-23]. HN1 has been proposed to act as a biomarker for diagnosis and prognosis of HCC [24]. In addition, previous work have also highlighted the associaltion between HN1 and DNA repair through bioinformatics analysis [24]. These observations intrigued us to further interrogate the multiple functions of HN1 in HCC, especially as a regulator of platinum drug sensitivity.

\section{Methods}

\section{Patients and cell lines}

We followed up 43 patients who were diagnosed as hepatocellular carcinoma through liver puncture and histopathology in the First Affiliated Hospital of Xi'an Jiaotong University. Subsequently, these patients received the oxaliplatin-based systemic chemotherapy. When the tumor was suppressed enough to correspond to surgical indications, hepatectomy was performed. Carcinoma tissues and para-carcinoma 
tissues (more than $3 \mathrm{~cm}$ distal to the edge of tumor) were collected from surgical specimens. The survival time of patients was followed up and recorded. All samples were collected from consenting individuals and on the basis of the protocol approved by the Ethics Committee of the First Affiliated Hospital of Xi'an Jiaotong University.

The human cell lines (MHCC-97L, MHCC-97H, HepG2, Hep3B, Huh7, SMCC-7721 and L02) were purchased from Cell Bank of the Chinese Academy of Sciences (Shanghai, China). All these cells were maintained in DMEM (HyClone, USA) with 10\% FBS (HyClone, USA) supplemented with $1 \%$ streptomycinpenicillin at $37^{\circ} \mathrm{C}$ and $5 \% \mathrm{CO} 2$.

\section{Transfection and lentiviral transduction}

All small interference RNAs (siRNA) and plasmids transfected into HepG2, MHCC-97L, Huh7 and Hep3B cell lines were co-incubated with lipofectamine 2000 (Invitrogen, USA) according to the standard protocol. The total RNA and protein were extracted after transfection $48 \mathrm{~h}$ and $72 \mathrm{~h}$ respectively and detected by qRT-PCR and Western blot. The following siRNAs and plasmids were designed and synthesized by Genepharma (Shanghai, China): 5'-UUCUCCGAACGUGUCACGUTT-3' for negative control siRNA; 5'GGAAGACUUGGAGUCAUCUTT-3' for siHN1\#1; 5'-CCGGAGACUUCUUAGAUCUTT-3' for siHN1\#2; 5'GCAUGAACCCCUUGUGCUG-3' for siTRIM28, 5'-GATGGTTGAATGAGCGTCATT-3' for siMAGEA3/6, 5'CCUCUUCCACUUUGUACUUTT-3' for siMAGEC2. The overexpression plasmid for HN1 and HMGB1, the lentivirus with a short hairpin RNA (shRNA) against human HN1 on the basis of the sequence of siHN1\#2 were all obtained from GenePharma. The stably transfected cells were selected by puromycin ( 2.5 $\mu \mathrm{g} / \mathrm{mL}$ ). Negative control cells were treated with the lentiviral scramble control shRNA.

\section{Western blot}

For isolation of total protein fractions, cells were lysed using RIPA lysis buffer containing proteinase inhibitor and phosphatase inhibitor. Total Protein lysate was separated by electrophoresis in SDSpolyacrylamide gels and transferred to PVDF membrane (Merck Millipore) and blocked by $5 \%$ milk at room temperature for 1 hour and then incubated with indicated primary antibodies at $4^{\circ} \mathrm{C}$ overnight. After incubated with secondary antibodies, the bands were visualized with chemiluminescence Imaging system by using the ECL immunoblotting kit (Millipore, USA). The band intensities were quantified by Image J software. The primary antibodies to HN1 (\#ab126705), gamma H2A.X (phosphor S139) (\#ab81299), Histone H4 (\#acetyl K5 + K8 + K12 + K16) (\#ab177790), Cyclin D1 (\#ab40754), MAGEA3 (\#ab223162) were obtained from Abcam. The antibodies to TRIM28 (\#sc-515790) and Histone H3 (acetyl k9 + k14) (\#sc-518011) were obtained from Santa Cruz Biotechnology. The antibodies to HMGB1 (\#10829-1-AP), c-Myc (\#10828-1-AP), ubiquitin (\#10201-2-AP), GAPDH (\#10494-1-AP), Ki67 (\#27309-1AP) were purchased from Proteintech. The antibodies to ZEB1, E-cadherin and N-cadherin (Kit \#9782) were purchased from Cell Signaling Technology.

\section{Quantitative real-time PCR (qRT-PCR)}


Total RNA was extracted using Trizol reagent (Invitrogen, USA) according to the manufacturer's protocol. Then the cDNA was obtained by using EasyQuick RT MasterMix kit (cw2019s, CWBIO, China). Then qRTPCR was performed using CFX384 Real Time System (BioRad) and SYBR Green PCR Master Mix (GenePharma). The expression level of GAPDH was endogenous control to normalize that of target genes. The 2- $\Delta \Delta \mathrm{Ct}$ method was calculated to compare the corresponding gene expression level. The primers were presented as followed: human HN1: 5'-ATAGCTCCCGAGTTTTGCGG-3' (forward), 5'GCCACCACTAGACTTGGCAC-3' (reverse); human HMGB1: 5'-GGGTACTGCCTTGCTTGACA-3' (forward), 5'TCCCAAGTTGTGGCAAGTGT-3' (reverse); human GAPDH: 5'-TCCTGCACCACCAACTGCTT-3' (forward); 5'GAGGGGGCCATCCACGTCTT-3' (reverse).

\section{Immunofluorescence}

Cells were seeded on glass coverslips to confluence,fixed in methylalcohollpermeabilized with $0.1 \%$ Triton X-100 diluted in PBS for 5 minutes and blocked by $10 \%$ goat serum (Sigma-Aldrich). Then cells were incubated with needed primary antibodies at $4^{\circ} \mathrm{C}$ overnight and then secondary antibodies being in tune with the manufacturer's introduction. The cells were stained by DAPI to highlight nucleus for $15 \mathrm{~min}$ at room temperature and finally imaged with Fluorescence confocal microscope.

\section{Immunohistochemistry}

HCC tissues of patients and nude mice were fixed in $4 \%$ paraformaldehyde for at least 48 hours, and were dehydrated and embed in paraffin. Tissues were cut into sections for immunohistochemistry staining. After deparaffinization with xylene, sections were subjected to antigen repair twice for 10 minutes at $100^{\circ} \mathrm{C}$ in citrate buffer $(\mathrm{pH}=6.0)$. Tissue section were blocked by $10 \%$ goat serum for 20 minutes and incubated with diluted primary antibody overnight at $4^{\circ} \mathrm{C}$. After being washed by phosphate buffer saline (PBS) for 3 times, tissue sections were incubated with the avidin-biotin complex for 12 minutes and were subjected to reaction with substrate of diaminobenzidine for appropriate time. Finally, the tissue sections were counterstained with hematoxylin. The immunoreactive score (IRS) was assessed according to the proportion of positive cells (no positive cells as $0 ;<10 \%$ positive cells as $1 ; 11-50 \%$ positive cells as 2 ; $51-80 \%$ positive cells as 3 ; and $>80 \%$ positive cells as 4 ) in the bright fields with 200 times magnification.

\section{Flow cytometry}

The degree of apoptosis was assessed by PE Annexin V Apoptosis Detection Kit I (BD Pharmingen) followed by flow cytometric analysis. The cells were harvested and washed three times with pre-cooling PBS. Within $100 \mu \mathrm{L}$ cell suspension at the density of $1 \times 10^{6}$ cells $/ \mathrm{mL}$ resuspended in $1 \times$ binding buffer, $5 \mu \mathrm{L} 7 \mathrm{AAD}$ and $5 \mu \mathrm{L}$ PE Annexin $\vee$ were added and incubated for 15 minutes kept out of light. Then $400 \mu \mathrm{L}$ $1 \times$ binding buffer was added to terminate the reaction and analysis was conducted by FACS.

\section{Cell proliferation and migration/invasion assays}

Colony formation assay and MTT assay were performed to detect cell proliferation potency. Cells transfected with siRNA or plasmid were seeded onto 6-well plates at a density of 500 cells/well. After two 
weeks, when a certain well appeared colonies containing more than 50 cells, the colonies were fixed with $5 \%$ methanol, stained with $0.1 \%$ crystal violet and finally counted. MTT assay is the assay that 1000 cells which were seeded on 96 -well plates per well and cultured for $24,48,72,96$ hours respectively, then treated with $0.5 \mathrm{mg} / \mathrm{mL} \mathrm{MTT}$, incubated for 4 hours at $37^{\circ} \mathrm{C}$, added $150 \mu \mathrm{l}$ DMSO for $10 \mathrm{~min}$ and finally followed by measured at 560nm absorbance using the EnSpire Multimode Plate Reader (PerkinElmer). Transwell assay was conducted to assess cell migration and invasion. To measure cell invasion, $5 \times 10^{4}$ cells resuspended in serum-free medium were seeded into the upper chamber of $8 \mu \mathrm{m}$ pore size insert (Costar, Corning, Cambridge, MA, USA) pre-coated with Matrigel (BD Biosciences) and DMEM medium with $20 \%$ FBS was added to the lower chamber. For migration, the same procedures were carried out except for the pro-treatment of Matrigel. After incubated at $37^{\circ} \mathrm{C}$ for 24 hours, cells on the bottom surface of chamber were fixed, stained and counted as the standard protocol.

\section{Cell viability and drug sensitivity}

$2 \times 10^{3}$ cells/well cells were cultured on $6 \mathrm{~cm}$ plates, added into drug and incubated for 24 hours as required at $37^{\circ} \mathrm{C}$. The CCK 8 kit (Dojindo Laboratories) was employed to measure cell viability and reflect drug sensitivity according to manufacturer's protocol. The absorbance at $450 \mathrm{~nm}$ was measured.

\section{Co-immunoprecipitation analysis}

Cells were lysed in NP40 buffer containing the protease inhibitor. After added into the specific antibodies and incubated at $4^{\circ} \mathrm{C}$ for 4 hours, cell lysis and activated protein A/G Plus-agarose beads (Santa Cruz Biotechnology, Inc., Santa Cruz, CA, USA) were co-incubated at $4^{\circ} \mathrm{C}$ overnight. The agarose beads were washed using RIPA for three times, added into SDS-PAGE loading buffer and immune complexes were subjected to subsequent western blot analysis.

\section{Alkaline Comet assay}

Alkaline comet assay is a sensitive genotoxicity tool typically for identification of DNA lesions. Cells were treated by oxaliplatin before embedded into $0.5 \%$ low melting point agarose gel in the single-cell suspension on clean slides precoated by $1.5 \%$ normal melting agarose. Then, cells protected from light undergone lysis for 1 hour at $4^{\circ} \mathrm{C}$, electrophoresis $(0.8 \mathrm{v} / \mathrm{cm}, 20$ minutes) and neutralization $(0.5 \mathrm{M}$ Tris$\mathrm{HCl}, \mathrm{pH}$ 7.5). Finally, cells were stained with GoldView and subjected to acquire images by fluorescence microscope. The cells with positive- tail were counted to measure DNA lesions.

\section{Transmission electron microscope (TEM)}

Cells were fixed in $2.5 \%$ glutaraldehyde for 5 minutes and collected. Then cell mass was placed in fresh $2.5 \%$ glutaraldehyde for at least 2 hours. After treated by $1 \%$ osmic acid for 1 hour, rinsed once again and then dehydrated with gradient concentration of acetone, cells were finally made out into ultrathin sections. The samples were observed under an $\mathrm{H}-7650$ transmission electron microscope (Hitachi, Tokyo, Japan).

\section{Tumor xenografts}


The laboratory animals used in this study were four-week-old male BALB/c nude mice raised in the Laboratory Animal Center (Xi'an Jiaotong University, China). The nude mice were randomly divided into 2 groups ( $n=7$ per group) and subcutaneously injected into inguina with MHCC-97L cells $\left(5 \times 10^{6}\right.$ in $200 \mu \mathrm{L}$ PBS) stably transfected with shHN1 or shNC. The sizes of tumor were measured every 3 days and all nude mice were sacrificed after 4 weeks when none of diameter of tumor exceeded $15 \mathrm{~mm}$. In lung metastasis model, above-mentioned cells $\left(1 \times 10^{6}\right.$ in $100 \mu \mathrm{L}$ PBS) were injected into the lateral tail vein of nude mice ( $n=3$ per group). Eight weeks later, the mice were sacrificed and lungs were stripped out. For testing the effect of HN1 on drug sensibility, the mice were divided randomly into two groups ( $n=8$ per group) and subcutaneously injected with MHCC-97L cells ( $1 \times 10^{7}$ in $200 \mu \mathrm{L}$ PBS) stably transfected with shHN1 and shNC respectively. When volume of some tumors roughly reached $100 \mathrm{~mm}^{3}(\mathrm{~V}=$ length $\times$ width $\left.^{2} \times 0.52\right)$, every group were randomly divided into two subgroups. The mice in experimental group were infected intraperitoneally with oxaliplatin $(10 \mathrm{mg} / \mathrm{kg})$ and the control groups with the same volume of normal saline (NS) every 3 days. After 24 days, the mice were sacrificed and stripped out of tumors. All animal experiments were approved by Animal Ethics Committee of Xi'an Jiaotong University.

\section{Statistics}

Data was statistically analyzed and graphed by GraphPad Prism 8 (GraphPad Software, San Diego, CA, USA). 2-tailed student's test was conducted for two groups and one-way ANOVA for multicomponent groups. Overall survival (OS) and progression free survival (PFS) were calculated by the Kaplain-Meier method and log-rank test on the basis of offered survival time. $p<0.05$ was supposed as statistically significant.

\section{Results}

\section{HN1 was highly expressed in HCC and associated with poor prognosis of HCC patients.}

First, the protein level of HN1 was analyzed by IHC in $43 \mathrm{HCC}$ tissues and para-carcinoma tissues, and the protein level of Ki67 was also analyzed in HCC tissues to assess cell proliferation of malignant cells (Fig. 1a). We found that HN1 protein level was higher and positively correlated with Ki67 protein level in HCC tissues with Pearson's correlation of 0.6498 (Fig. 1b). The overexpression of HN1 in HCC tissues compared to para-carcinoma tissues was verified by western blot (Fig. 1c). Moreover, the protein and mRNA expression level of HN1 in human HCC cell lines were remarkably higher than that in the normal immortalized hepatic cell line LO2 (Fig. 1d, e). Notedly, Hep3B and Huh7, which have been proved to be sensitive to oxaliplatin [25], had lower level of HN1 than other cell lines in our study. The clinical data indicated that aberrant expression of HN1 was closely associated with the tumor size and the incidence of portal vein tumor thrombus (PVTT) of HCC (see additional file 1). Further analysis of the overall survival and progression free survival of the patients showed that patients with higher expression of HN1 had worse prognosis than those who had lower expression of HN1 (Fig. 1f, g). Overall, above data implied that HN1 was overexpressed in HCC tissues and closely associated with poor prognosis of patients. 


\section{HN1 knockdown inhibited HCC proliferation and metastasis in vitro.}

To investigate the tumor-promoting mechanism of HN1 in HCC, two different siRNAs (siRNA\#1 and siRNA\#2) were transfected into HepG2 and MHCC-97L cell lines. The cells transfected with siHN1\#2 had a lower HN1 mRNA expression and protein level than siHN1\#1 (Fig. 2a, b). The MTT assay showed that downregulation of HN1 significantly inhibited HCC cell growth (Fig. 2c), and HN1 knockdown inhibited clonogenicity of HCC cells (Fig. 2d). In addition, c-Myc and cyclin D1 which reflect potency of cell proliferation also downregulated in HN1 knockdown cells (Fig. 2f). However, downregulation of HN1 had no effect on cell apoptosis (see additional file 2).

Given that higher expression of HN1 was related with PVTT patients, HN1 possibly promoted metastasis of HCC. As expected, the result of transwell assay showed that downregulation of HN1 decreased the migration and invasion of HCC cells (Fig. 2e). In addition, N-cadherin, E-cadherin and ZEB1 which reflect metastasis potency of HCC cells also altered following HN1 knockdown (Fig. 2g). Next, we overexpressed HN1 in Hep3B and Huh7 cell lines through pCMV-HN1 plasmid, and measured their potency of cell proliferation, migration and invasion. We found that these malignant phenotypes of HCC cells were consistently reinforced after HN1 overexpression (see additional file 3). In conclusion, gain and loss of function experiments indicated that HN1 could promote proliferation, migration and invasion of HCC cells.

\section{HN1 knockdown increased sensitivity to oxaliplatin and aggravated DNA damage in HCC cells.}

We established HN1 knockdown cells lines of HCC (HepG2 and MHCC-97L) through shRNA lentivirus vector and knockdown efficiency was confirmed by qRT-PCR and western blot (Fig. 3a, b). Taking the observation into account that patients with higher HN1 expression had worse prognosis after oxaliplatinbased chemotherapy treatment, we explored whether HN1 desensitized HCC to oxaliplatin. The CCK8 assay demonstrated that HepG2 and MHCC-97L cells with HN1 knockdown were more sensitive to oxaliplatin and had lower $\mathrm{IC}_{50}$ compared to those with negative control HCC cells (Fig. 3c). Alkaline comet assay suggested that HCC cells with HN1 knockdown had higher tail-positive proportion compared with negative control group, which indicated that there were more DNA lesions in HN1 knockdown HCC cells after oxaliplatin treatment (Fig. 3d). In addition, HN1 knockdown also reduced the survival of HCC cells in low dose of oxaliplatin ( $1 \mu \mathrm{M}$ and $5 \mu \mathrm{M}$, Fig. 3e). Next, we treated HCC cells with gradient concentration of oxaliplatin $(1 \mu \mathrm{M}, 5 \mu \mathrm{m}$ and $10 \mu \mathrm{M})$ for 1 hour or 24 hours (Fig. 3f, g). We measured the histone modification related to DNA damage $(\mathrm{\gamma}-\mathrm{H} 2 \mathrm{AX})$ and chromatin accessibility (acetylation of $\mathrm{H} 3$ and $\mathrm{H} 4$ ) [26-28]. We found that after oxaliplatin treatment, $\mathrm{Y}-\mathrm{H} 2 \mathrm{AX}$ level was higher in HN1 knockdown HCC cells while acetylation of $\mathrm{H} 3$ and $\mathrm{H} 4$ were higher in negative control HCC cells. Then time-dependence assay (cells treated with $5 \mu \mathrm{M}$ oxaliplatin for $0 \mathrm{~h}, 6 \mathrm{~h}, 12 \mathrm{~h}$ and $24 \mathrm{~h}$ ) were performed to verify that HN1 could affect DNA damage repair and chromatin modification (Fig. 3h). We also confirmed that immunofluorescence intensity of $\mathrm{y}-\mathrm{H} 2 \mathrm{AX}$ foci is higher in HN1 knockdown HCC cells after oxaliplatin treatment $(5 \mu \mathrm{M}$ and $10 \mu \mathrm{M}$, Fig. 3i). Finally, flow cytometry analysis demonstrated that HN1 knockdown HCC cells had a significantly higher apoptosis rate compared to negative control cells after oxaliplatin treatment $(5 \mu \mathrm{M}$ 
and $10 \mu \mathrm{M}$, Fig. 3j). All these results indicated that HN1 knockdown substantially sensitized HCC cells to oxaliplatin treatment.

\section{HN1 inhibited the degradation of HMGB1 through autophagy-lysosome pathway by interacting with TRIM28.}

Given that the depletion of HN1 in HCC cells enhanced sensitivity to oxaliplatin-induced DNA damage, we hypothesized that deregulation of proteins in DNA repair pathway could be crucial to this sensitivity transformation. HMGB1, which can bind to damaged DNA, recruit other components and enhance DNA repair, was tested as a potential downstream effector of HN1. The result of qRT-PCR showed that mRNA expression level of HMGB1 was impervious followed by HN1 knockdown (Fig. 4a). The protein expression level of intracellular HMGB1, however, was obviously decreased both in nucleus and cytoplasm (Fig. 4b, c). This result was verified by immunofluorescence staining (Fig. 4d). These results indicated that HN1 could regulate the expression of HMGB1 in post-transcriptional level and affect its degradation. To verify this speculation, after cells were transfected into PCMV-HMGB1 expression plasmid for 72 hours, the protein synthesis inhibitor, cycloheximide (CHX) was added at a concentration of $2 \mu \mathrm{g} / \mathrm{mL}$ for another 24 hours, 48 hours, 72 hours, and 96 hours respectively. The result of western blot showed that degradation of HMGB1 is prominently faster in HN1 knockdown cells than negative control cells (Fig. 4e). Next, we aimed to investigate which pathway was responsible for HN1-related HMGB1 degradation. The autophagy inhibitor, chloroquine $(10 \mu \mathrm{M})$ or proteasome inhibitor, MG132 $(1 \mu \mathrm{M})$ was individually added into cells for 24 hours in the presence of $\mathrm{CHX}(2 \mu \mathrm{g} / \mathrm{mL})$. The result of western blot showed that autophagy inhibitor chloroquine could slower evidently the degradation rate of HMGB1 in HN1 knockdown cells (Fig. 4f). Ubiquitination is a common post-translational modification for substrate protein ready to be degraded [29]. Thus, we detected the endogenous ubiquitination of HMGB1. The result of IP showed that ubiquitination of HMGB1 was substantially increased followed by HN1 knockdown (Fig. 4g). These results indicated that HN1 prevent HMGB1 from autophagy-lysosome-depend degradation.

We next investigate how HN1 regulated the ubiquitination of HMGB1. Previous studies reported that TRIM28 could be essential to the ubiquitination and degradation of HMGB1 [30]. The result of coimmunoprecipitation assay revealed that HN1 interacted with TRIM28 (Fig. 4h), which is also supported by STRING database (https://string-db.org). In addition, from analysis of Immunofluorescence colocalization assay, we found the high degree of co-localization of HN1 and TRIM28 protein (Fig. 4i). Our pre-test indicated that TRIM28 knockdown could slightly affect the protein level of HMGB1 in the normal or HN1 knockdown cells (see additional file 4).

It has been well-established that MAGE proteins can bind to RING domain of E3 ligase like TRIM28 and form cancer-specific ubiquitin ligase complexes which regulate ubiquitination and degradation of various tumor suppressors like p53 [30-32]. It's worth noting that MAGE-C2 and MAGE-A3/ 6 was specially upregulated in HCC [33-35]. Therefore, we hypothesized that MAGE-C2 or (and) MAGE-A3/6 could be involved in regulation of HMGB1 ubiquitination through TRIM28. We then knockdown mRNA expression 
of TRIM28, MAGE-A3/6 or MAGE-C2 with small interfering RNAs (siRNAs), and detected the protein level of HMGB1. We found that knockdown of TRIM28 and MAGE-A3/6 significantly restored the protein level of HMGB1, while knockdown of MAGE-C2 had no effect on HMGB1 protein level (Fig. 4j). As expected, knockdown of TRIM28 or MAGE-A3/6 could inhibit the ubiquitination of HMGB1 and restored its protein level in HCC cells with HN1 downregulation (Fig. 4k). Based on these observations, we drawn the conclusion that HN1 could inhibit the degradation of HMGB1 through autophagy-lysosome pathway via interacting with TRIM28-MAGE-A3/6 complexes.

\section{Overexpression of HMGB1 restored the phenotypes resulted from HN1 knockdown in HCC cells.}

To further investigate the essential roles of HMGB1 as a key mediator of HN1 in phenotypic regulation of HCC cells, we transfected pCMV-HMGB1 overexpression plasmid into HN1 knockdown cell lines and assessed their phenotype restoration. We confirmed the transfection efficiency of plasmid through qRTPCR and western blot analysis (Fig. 5a, e). The results of MTT assay and clone formation assay showed that the potency of cell growth could be partly rescued by overexpression of HMGB1 in HN1 knockdown cells and so was potency of migration and invasion tested by Transwell assay (Fig. 5b-d). In addition, results of western blot showed that HMGB1 overexpression could partly reverse the protein expression level of the biomarkers of cell proliferation and metastasis in HepG2 and MHCC-97L cells (Fig. 5e). Finally, we observed that overexpression of HMGB1 could abolish the increased drug sensitivity induced by HN1 knockdown in HCC cells (Fig. 5f). The results of western blot revealed that DNA damage alleviated and chromatin accessibility increased followed by HMGB1 overexpression under oxaliplatin treatment (Fig. 5g).

Moreover, increasing evidences indicated that autophagy plays an essential role in regulation of malignant phenotypes of tumor and cancer resistance to chemotherapy agents. Given that HMGB1 is an acknowledged autophagy activator [36], we conjectured that HN1 could regulate autophagy via HMGB1 and further affect phenotypes of HCC cells. In contrast to shNC cells, the recognized autophagy stimuli

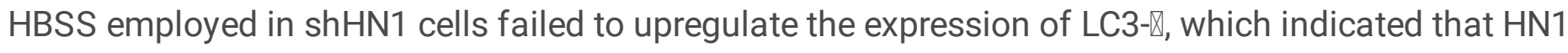
was essential to cell autophagy (Fig. 5h). To investigate the role of HMGB1 in this process, we transfected pCMV-HMGB1 overexpression plasmid into shNC and shHN1 HCC cells and results of western blot indicated that the overexpression of HMGB1 boosted the protein level of LC3-邓and lowered the level of p62 in shHN1 cells (Fig. 5i). In addition, cells were transfected by exogenous GFP-mRFP-LC3 adenovirus to monitor and measure cell autophagy. We found that the formation of GFP-mRFP-LC3 puncta markedly increased in shHN1 cells with HMGB1 overexpression compared with single shHN1 cells (Fig. 5j). Finally, transmission electron microscope analysis provided potent testimony of autophagy. The overexpression of HMGB1 in shHN1 cells exhibited significantly more autophagosomes compared with single HN1 knockdown cells (Fig. 5k). Above evidences supported a pivotal role of HMGB1 in process of autophagy regulated by HN1.

The above results verified that HMGB1 acted as a key downstream effector of HN1 in regulation of malignant phenotypes of HCC cells such like cell proliferation, metastasis and chemosensitivity. 
HN1 knockdown inhibited malignant phenotypes and enhanced the antitumor effect of oxaliplatin in vivo.

MHCC-97L cells stably transfected with shNC or shHN1 were subcutaneously injected into male nude mice. We found that knockdown of HN1 dramatically inhibited tumor growth compared with negative control group (Fig. 6a-c). From the result of IHC, we also found the protein level of Ki67 and HMGB1 were also decreased after HN1 knockdown (Fig. 6d). Additionally, the role of HN1 on HCC tumor metastasis was evaluated through caudal intravenous pulmonary metastasis model. The result of HE staining showed that HN1 knockdown reduced the number and size of visible pulmonary metastatic nodules and the Ki67 protein level in metastatic niche of lung (Fig. 6e, f). Briefly, HN1 knockdown inhibited tumor proliferation and metastasis in vivo. Furthermore, to investigate the effect of HN1 knockdown on HCC sensitivity to oxaliplatin treatment in vivo, the nude mice were treated with oxaliplatin or normal saline (NS) when some of tumors derived from HN1 knockdown or negative control MHCC-97I cells grew roughly to $100 \mathrm{~mm}^{3}$. The growth curve, image and weight of tumor demonstrated that HN1 knockdown group was more sensitive to oxaliplatin treatment than the control group (Fig. $6 \mathrm{~g}-\mathrm{i}$ ).

\section{Discussions}

HN1 reportedly promoted proliferation and metastasis of malignant cells in many human cancers. Recent research revealed that HN1 could increase STMN1 protein level by inhibiting its ubiquitination-mediated degradation to promote aggressiveness of anaplastic thyroid carcinoma [21]. In addition, previous work demonstrated that ectopic HN1 expression could increase degradation of $\beta$-catenin and resulted in disassociation of $\beta$-catenin and E-cadherin, which promoted progression of prostate cancer [22]. In our study, HMGB1 acted as an essential downstream target of HN1 to affect HCC cells malignant phenotypes. Similar to above-mentioned recognized mechanism, HN1 protected HMGB1 from the ubiquitination and subsequent degradation. Also, Chen Zhang and colleagues proved that HN1 could promote migration and invasion of breast cancer by enhancing the protein level of c-Myc and its target genes [23]. Consistently, we also found that HN1 could inhibit ubiquitination of endogenous c-Myc (see additional file 5). Base on above findings, we could reach a conclusion that HN1 possibly play an extensively regulatory role at the post-translation level. Our data further indicated that TRIM28 acted as a crucial mediator in this process. However, whether TRIM28 is a common or specific component to mediate oncoprotein degradation in context of HCC need more research.

Intranuclear HMGB1 mainly functions as DNA chaperone and can also be released into cytoplasm or extracellular context in response to diverse stimulus (eg, chemoradiotherapy and metabolic stress). Whether extracellular or intracellular HMGB1 has been proved to function as a crucial regulator of autophagy [36-39]. Here our study focused on intracellular HMGB1. The overwhelming evidences support that autophagy is important for cancer cells to survive from treatment of DNA damaging agents and ionizing radiation [40]. Indeed, we found that downregulation of HMGB1 followed by HN1 knockdown resulted in the suppression of autophagy, which was parallel with the enhancement of drug sensitivity. Another interesting bit of finding is that the regulatory mechanism of HN1-mediated HMGB1 degradation was dependent on autophagy-lysosome pathway. On the one hand, HN1 could inhibit the degradation of 
HMGB1 via the autophagy-lysosome pathway through interacting with TRIM28. On the other hand, HMGB1 in turn acted as the pivotal mediator of HN1 to promote autophagy in HCC cells. The mainstream view believes that unneeded cellular components can conduct selective autophagy to undergo precisely regulated and specific degradation pathway[41]. Selective autophagic receptors and cargo labeled by ubiquitin are indispensable for this process[41]. Our finding indicated that the degradation of specific molecule via autophagy-lysosome pathway was much more complex, subtle and context dependent.

TRIM28, a member of transcriptional intermediary factor 1 (TIF1) family, is a highly complex chromatinbinding protein with multiple domains structure and enzymatic activities [42]. Increasing findings suggested that TRIM28 could function to affect genome stability by involvement in the chromatin relaxation, chromatin-context response to DNA damage and cell cycle regulation [43]. Studies found that TRIM28 was significantly upregulated across various cancers, such as glioma [44], ovarian [45], breast [46] and gastric cancer [47] and was associated with the dismal overall survival of these cancer patients. However, TRIM28 played dual roles of tumor promotor or suppressor in context of HCC where it was highly expressed compared to normal counterparts and predicted the worse prognosis, whereas it acted as a tumor suppressor to prevent tumorigenic conversion in murine HCC model [48, 49].In our present research, ubiquitination of HMGB1 decreased and HMGB1 protein level restored prominently after TRIM28 knockdown in the HN1 knockdown cells. In addition, we found TRIM28 could interact with HN1. Based on these observations, we concluded that highly expressed HN1 probably sheltered the E3 ligase activity of TRIM28 through interacting with TRIM28 and inhibited degradation of HMGB1. MAGE proteins have been verified as the crucial function facilitator of E3 ligase activity of TRIM28 [31]. Conversely, our findings indicated that HN1 acted as the function suppressor of TRIM28 in HCC cells. Maybe HN1 and MAGE are like two ends of a seesaw of TRIM28 function, and TRIM28 itself has no preference for being a promoter or a suppressor of cancer progression unless it associates with some certain specific proteins like MAGE proteins or HN1. Given that TRIM28 serves as a large scaffold protein, whether there are other specific proteins that can coordinate biological activity of TRIM28 need further exploration.

\section{Conclusions}

In conclusion, we found that HN1 expression promoted malignant phenotypes of HCC cells and desensitized them to oxaliplatin treatment. Mechanically, HN1 inhibited the degradation of HMGB1 protein through autophagy lysosome pathway via interacting with TRIM28. Due to the difference in subcellular distribution, HMGB1 exerted both intranuclear and cytoplasmic mechanisms to meditate the biological effects of HN1. For the intranuclear mechanism, downregulation of HMGB1 caused by HN1 knockdown increased DNA damage and cell death. For the cytoplasmic mechanism, HN1 reduced the chemotherapy sensitivity through enhancing autophagy by stabilizing HMGB1 protein. So far, we nearcomprehensively explored the biological effects of HN1 in HCC. HN1 will be a promising intervention target to treat HCC, especially chemo-resistant cases for better prognosis.

\section{Abbreviations}


HCC: Hepatocellular carcinoma; $\mathrm{IC}_{50}$ : Half-maximal inhibitory concentrations; IHC:

Immunohistochemistry; HN1: Hematological and neurological expressed 1; HMGB1: High mobility group protein B1; NC: Negative control; shRNA: short hairpin RN; siRNA: small interference RNA

\section{Declarations}

\section{Acknowledgments}

We thank Translational Medicine Center, the First Affiliated Hospital of Xi 'an Jiaotong University for laboratory support. We thank the Laboratory Animal Center, Xi'an Jiaotong University to provide animal feeding room. We thank the 43 patients with HCC from the First Affiliated Hospital of Xi 'an Jiaotong University to provide clinical data and samples.

\section{Author's contributions}

$\mathrm{RHW}$ contributed significantly to the design, implement of the most of experiment and the draft of the manuscript. YNF contributed greatly to the implement of in vivo experiment and IHC analysis. MLY and YMC assistted in some cell experiments. YZ and XLL contributed to collection and analysis of patient data. YRL and SXH provided material and technical support and participated in ultimate evaluation of entirety of project in all rounds of manuscript editing. All authors read and approved the final manuscript.

\section{Funding}

The work was supported by grants from the National Natural Science Funds of China (Grant No.81502095) and Institutional Foundation of the First Affiliated Hospital of Xi'an Jiaotong University (No. 2020QN-12)

\section{Availability of data and materials}

All data generated and/or analyzed during this study are included in this article and its supplementary information files.

\section{Ethics approval and consent to participate}

This study was approved by the Ethics Committee of the First Affiliated Hospital of Xi'an Jiao tong University. Informed consent was obtained from the patients or their guardians. All animal experiments were approved by Animal Ethics Committee of Xi'an Jiaotong University.

\section{Consent for publication}

The authors declared their consent to submit the article for publication.

\section{Conflict of Interest}


The authors declared that they have no competing interests.

\section{References}

1. Sung, $\mathrm{H}$., et al., Global cancer statistics 2020: GLOBOCAN estimates of incidence and mortality worldwide for 36 cancers in 185 countries. 2021.

2. Lyu, N., et al., Hepatic arterial infusion of oxaliplatin plus fluorouracil/leucovorin vs. sorafenib for advanced hepatocellular carcinoma. 2018. 69(1): p. 60-69.

3. He, M., et al., Hepatic artery infusion chemotherapy using mFOLFOX versus transarterial chemoembolization for massive unresectable hepatocellular carcinoma: a prospective nonrandomized study. 2017. 36(1): p. 83.

4. Lytle, N.K., A.G. Barber, and T. Reya, Stem cell fate in cancer growth, progression and therapy resistance. Nat Rev Cancer, 2018. 18(11): p. 669-680.

5. Jung, Y. and S.J.C.r. Lippard, Direct cellular responses to platinum-induced DNA damage. 2007. 107(5): p. 1387-407.

6. Mukherjee, A. and K.M. Vasquez, Targeting Chromosomal Architectural HMGB Proteins Could Be the Next Frontier in Cancer Therapy. Cancer Res, 2020. 80(11): p. 2075-2082.

7. Mukherjee, A. and K.J.N.a.r. Vasquez, HMGB1 interacts with XPA to facilitate the processing of DNA interstrand crosslinks in human cells. 2016. 44(3): p. 1151-60.

8. Lange, S., D. Mitchell, and K.J.P.o.t.N.A.o.S.o.t.U.S.o.A. Vasquez, High mobility group protein B1 enhances DNA repair and chromatin modification after DNA damage. 2008. 105(30): p. 10320-5.

9. Yuan, F., et al., Evidence for involvement of HMGB1 protein in human DNA mismatch repair. 2004. 279(20): p. 20935-40.

10. Huang, H., et al., Hepatocyte-specific high-mobility group box 1 deletion worsens the injury in liver ischemia/reperfusion: a role for intracellular high-mobility group box 1 in cellular protection. 2014. 59(5): p. 1984-1997.

11. Ito, $\mathrm{H}$., et al., HMGB1 facilitates repair of mitochondrial DNA damage and extends the lifespan of mutant ataxin-1 knock-in mice. 2015. 7(1): p. 78-101.

12. Yang, M., et al., Poly-ADP-ribosylation of HMGB1 regulates TNFSF10/TRAIL resistance through autophagy. 2015. 11(2): p. 214-24.

13. Liu, K., et al., MIR34A regulates autophagy and apoptosis by targeting HMGB1 in the retinoblastoma cell. 2014. 10(3): p. 442-52.

14. Huang, J., et al., Targeting HMGB1-mediated autophagy as a novel therapeutic strategy for osteosarcoma. 2012. 8(2): p. 275-7.

15. Lv, D., et al., HMGB1 Promotes Prostate Cancer Development and Metastasis by Interacting with Brahma-Related Gene 1 and Activating the Akt Signaling Pathway. 2019. 9(18): p. 5166-5182.

16. Yan, W., et al., High-mobility group box 1 activates caspase-1 and promotes hepatocellular carcinoma invasiveness and metastases. 2012. 55(6): p. 1863-75. 
17. Nehil, M., et al., High mobility group box 1 promotes tumor cell migration through epigenetic silencing of semaphorin 3A. 2014. 33(44): p. 5151-62.

18. Zhou, H., et al., Cannabinoid receptor 2 promotes the intracellular degradation of HMGB1 via the autophagy-lysosome pathway in macrophage. 2020. 78: p. 106007.

19. Wu, J., et al., CMA down-regulates $p 53$ expression through degradation of HMGB1 protein to inhibit irradiation-triggered apoptosis in hepatocellular carcinoma. 2017. 23(13): p. 2308-2317.

20. Zhou, G., et al., Cloning, expression and subcellular localization of HN1 and HN1L genes, as well as characterization of their orthologs, defining an evolutionarily conserved gene family. 2004. 331: p. 115-23.

21. Pan, Z., et al., HN1 promotes tumor growth and metastasis of anaplastic thyroid carcinoma by interacting with STMN1. 2021. 501: p. 31-42.

22. Varisli, L., et al., HN1 negatively influences the $\beta$-catenin/E-cadherin interaction, and contributes to migration in prostate cells. 2015. 116(1): p. 170-8.

23. Zhang, C., et al., HN1 contributes to migration, invasion, and tumorigenesis of breast cancer by enhancing MYC activity. 2017. 16(1): p. 90.

24. Liu, Z., et al., HN1 as a diagnostic and prognostic biomarker for liver cancer. 2020. 40(7).

25. Martin, S., et al., PKM2 inhibition may reverse therapeutic resistance to transarterial chemoembolization in hepatocellular carcinoma. 2020. 39(1): p. 99.

26. Bonner, W., et al., GammaH2AX and cancer. 2008. 8(12): p. 957-67.

27. Kinner, A., et al., Gamma-H2AX in recognition and signaling of DNA double-strand breaks in the context of chromatin. 2008. 36(17): p. 5678-94.

28. Ramanathan, B. and M.J.C. Smerdon, Changes in nuclear protein acetylation in u.v.-damaged human cells. 1986. 7(7): p. 1087-94.

29. Dikic, I.J.A.r.o.b., Proteasomal and Autophagic Degradation Systems. 2017. 86: p. 193-224.

30. Pineda, C., et al., Degradation of AMPK by a cancer-specific ubiquitin ligase. 2015. 160(4): p. 715728.

31. Doyle, J., et al., MAGE-RING protein complexes comprise a family of E3 ubiquitin ligases. 2010. 39(6): p. 963-74.

32. Jin, J., et al., Sequential ubiquitination of p53 by TRIM28, RLIM, and MDM2 in lung tumorigenesis. 2020.

33. Riener, M., et al., Frequent expression of the novel cancer testis antigen MAGE-C2/CT-10 in hepatocellular carcinoma. 2009. 124(2): p. 352-7.

34. Tahara, K., et al., Expression of the MAGE gene family in human hepatocellular carcinoma. 1999. 85(6): p. 1234-40.

35. Wang, C., B. Lin, and C.J.I.j.o.c. Chen, An aptamer targeting shared tumor-specific peptide antigen of MAGE-A3 in multiple cancers. 2016. 138(4): p. 918-26.

36. Tang, D., et al., Endogenous HMGB1 regulates autophagy. 2010. 190(5): p. 881-92. 
37. Wang, S., Y.J.J.o.h. Zhang, and oncology, HMGB1 in inflammation and cancer. 2020. 13(1): p. 116.

38. Livesey, K., et al., p53/HMGB1 complexes regulate autophagy and apoptosis. 2012. 72(8): p. 19962005.

39. Tang, D., et al., The redox protein HMGB1 regulates cell death and survival in cancer treatment. 2010. 6(8): p. 1181-3.

40. Chang, H., Z.J.J.o.h. Zou, and oncology, Targeting autophagy to overcome drug resistance: further developments. 2020. 13(1): p. 159.

41. Gatica, D., V. Lahiri, and D.J.N.c.b. Klionsky, Cargo recognition and degradation by selective autophagy. 2018. 20(3): p. 233-242.

42. Hatakeyama, S.J.N.r.C., TRIM proteins and cancer. 2011. 11(11): p. 792-804.

43. McAvera, R. and L.J.C. Crawford, TIF1 Proteins in Genome Stability and Cancer. 2020. 12(8).

44. Su, C., et al., TRIM28 is overexpressed in glioma and associated with tumor progression. 2018. 11: $\mathrm{p}$. 6447-6458.

45. Cui, Y., et al., High levels of KAP1 expression are associated with aggressive clinical features in ovarian cancer. 2014. 16(1): p. 363-77.

46. Addison, J., et al., KAP1 promotes proliferation and metastatic progression of breast cancer cells. 2015. 75(2): p. 344-55.

47. Yokoe, T., et al., KAP1 is associated with peritoneal carcinomatosis in gastric cancer. 2010. 17(3): p. 821-8.

48. Wang, Y., et al., KAP1 is overexpressed in hepatocellular carcinoma and its clinical significance. 2016. 21(5): p. 927-933.

49. Herquel, B., et al., Transcription cofactors TRIM24, TRIM28, and TRIM33 associate to form regulatory complexes that suppress murine hepatocellular carcinoma. 2011. 108(20): p. 8212-7.

\section{Figures}


a.

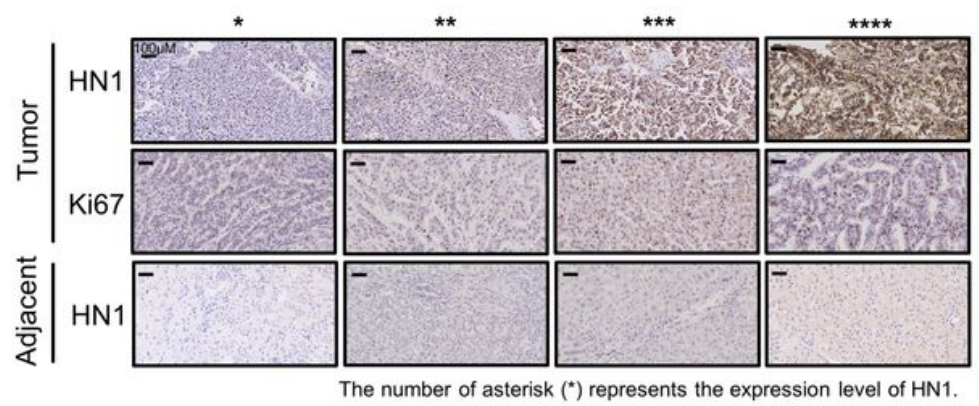

C.

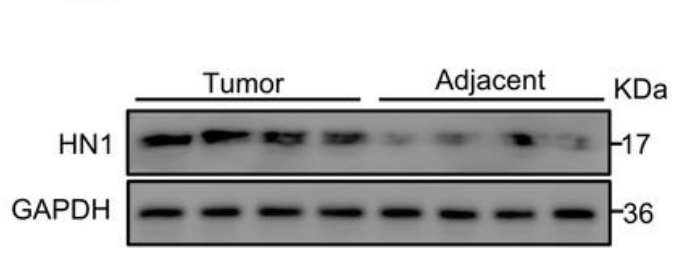

f.

Overall survival

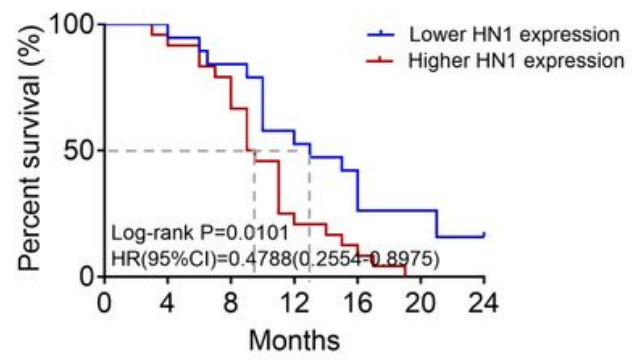

g. b.

Correlation of $\mathrm{HN} 1$ and $\mathrm{Ki} 67$ in $\mathrm{HCC}$

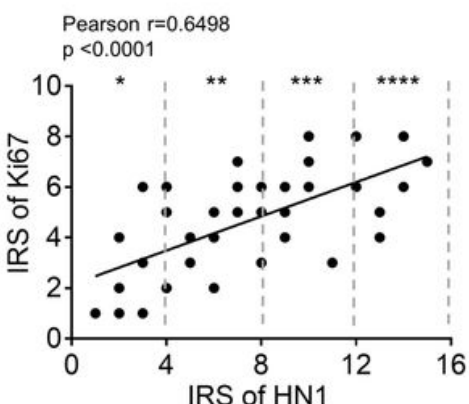

e.

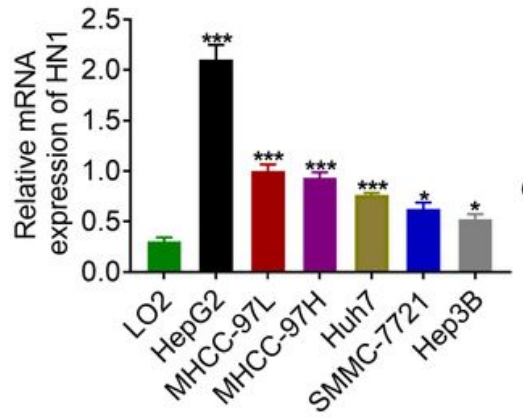

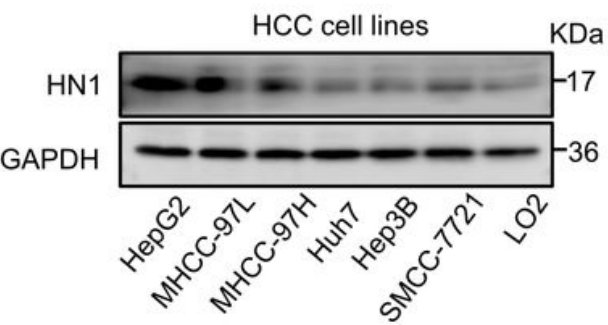

Progression free survival

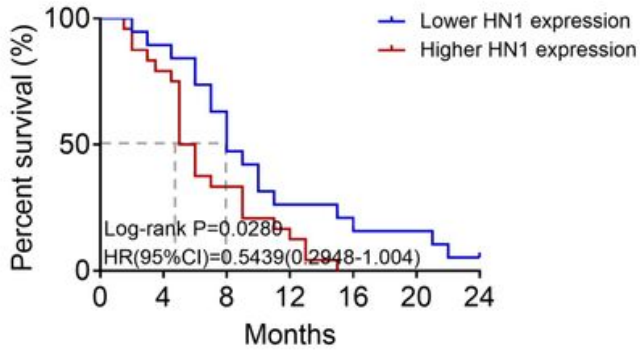

Figure 1

HN1 was highly expressed in $\mathrm{HCC}$ and associated with poor prognosis of HCC patients. (a) Immunohistochemistry staining of HN1 and Ki67 in HCC and adjacent para-carcinoma tissues (200xmagnification). (b) Correlation of HN1 and Ki67 protein level of HCC. $p<0.0001$. (c) Western blot analysis of HN1 protein level of HCC tissues. (d, e) qRT-PCR and western blot analysis of HN1 expression in the immortalized normal human LO2 cell line and HCC cell lines. (f, g) Kaplan-Meier analysis of OS and PFS based on HN1 expression level in HCC patients treated with oxaliplatin-based chemotherapy. 
a.

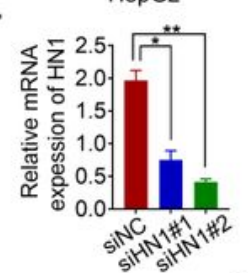

C.

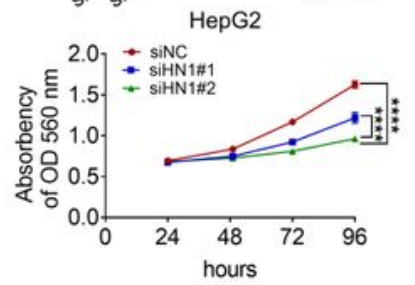

d.

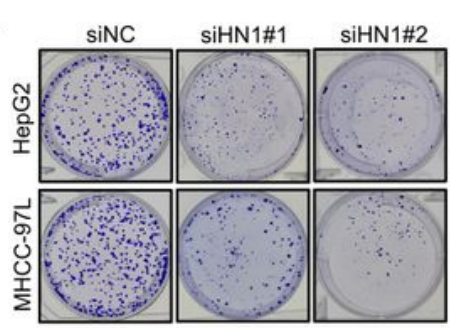

e.
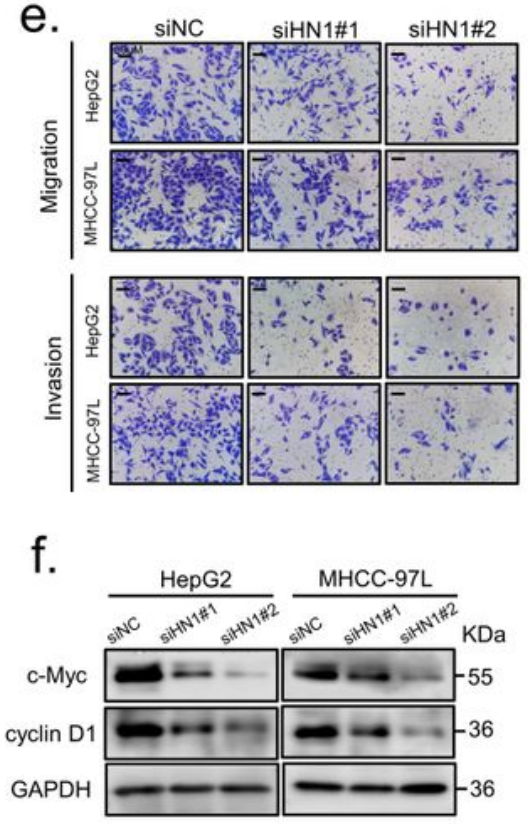

b.

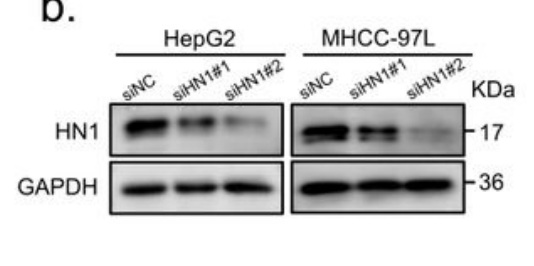

MHCC-97L

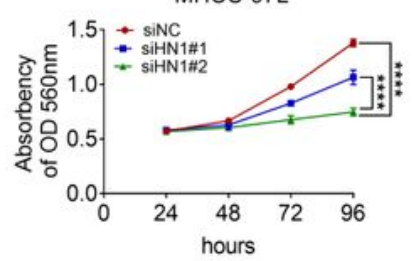

HepG2
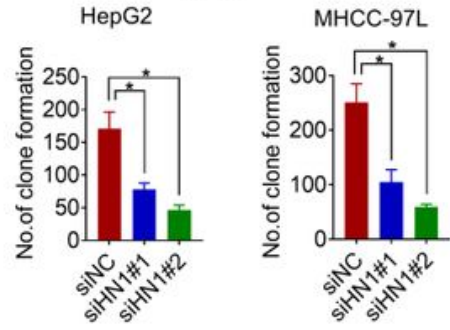

HepG2

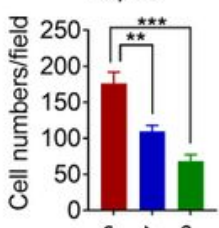

(c)

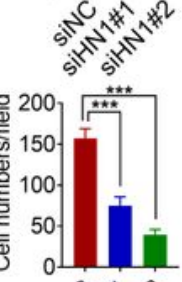

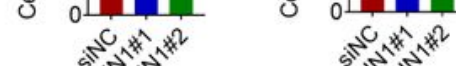

g.
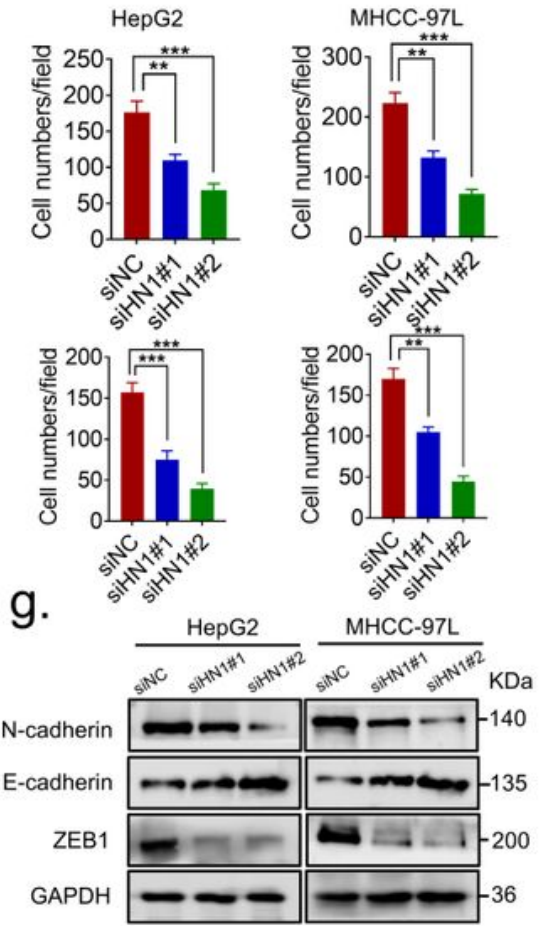

\section{Figure 2}

HN1 knockdown inhibited HCC proliferation and metastasis in vitro. $(a, b)$ qRT-PCR and western blot analysis of HN1 expression after cells were transfected into two different HN1siRNAs. ${ }^{*} p<0.05,{ }^{* \star} p<0.01$. (c, d) Cell proliferation potency after HN1 knockdown was tested by MTT assay and clone formation assay. ${ }^{*} p<0.05,{ }^{*} * \star x p<0.0001$. (e) The migration and invasion potency of HCC cells after HN1 knockdown was tested by Transwell assay (400* magnification). ${ }^{*} \mathrm{p}<0.01,{ }^{* * *} \mathrm{p}<0.001$. (f, g) Protein 
levels of representative proliferation and metastasis biomarkers after HN1 knockdown were analyzed by western blot.
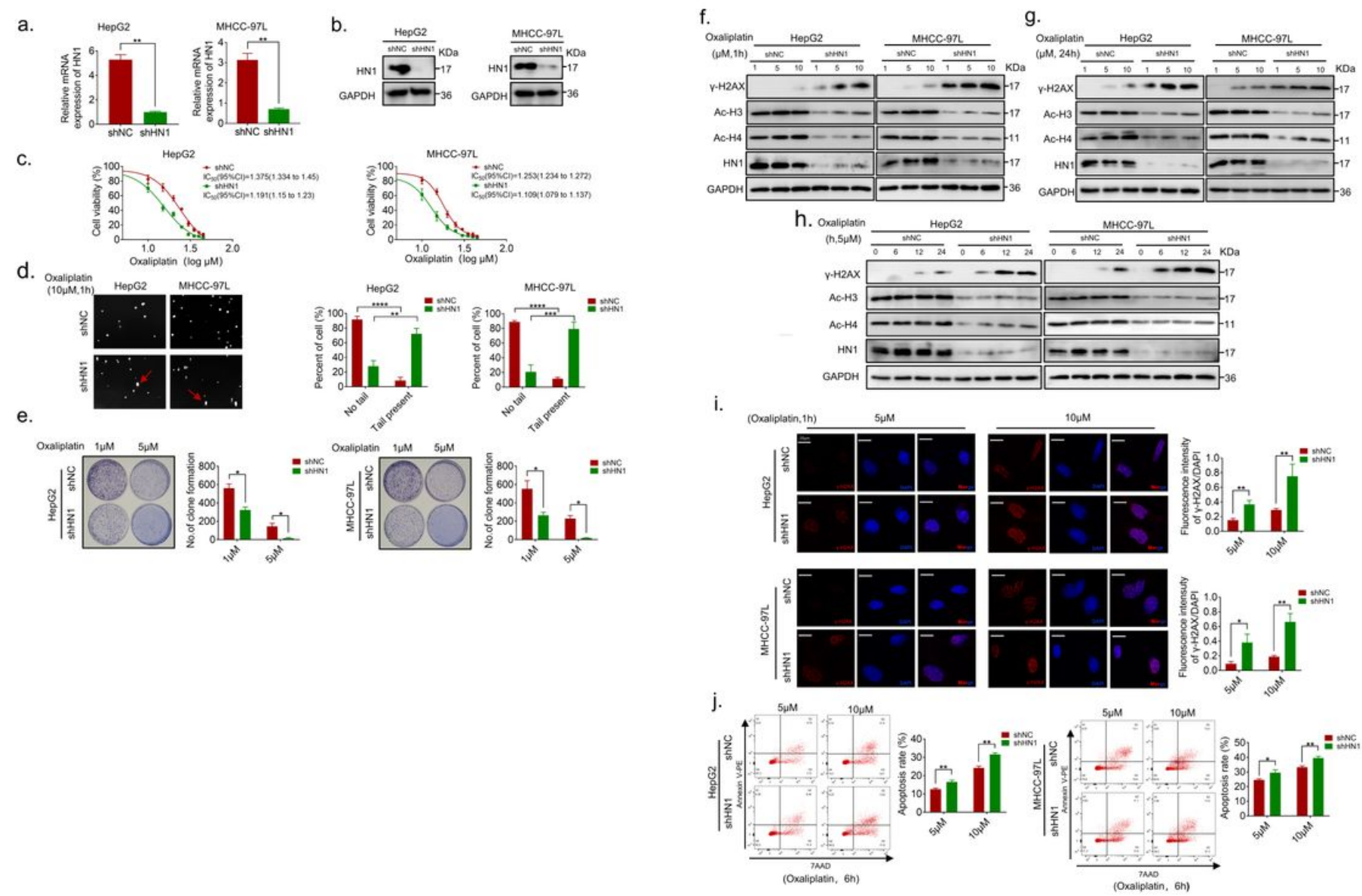

Figure 3

HN1 knockdown increased sensitivity to oxaliplatin and aggravated DNA damage in HCC cells. (a, b) qRTPCR and western blot analysis of HN1 expression after cells were transfected into shHN1 and shNC. (c) Cell viability was tested by CCK8 assay to compare HN1 knockdown cells with negative control cells after treatment of oxaliplatin. HN1 knockdown sensitized HCC cells to oxaliplatin treatment. ${ }^{* \star \star \star} p<0.0001$. The data was subjected to non-linear regression $\left(Y=\right.$ Bottom + (Top - Bottom) $/\left(1+10^{\wedge}(\right.$ LoglC50 $\left.-X)\right)$ and IC50 of each group was calculated by variable slope model. (d) Alkaline comet assay was performed to observe the DNA lesions of HCC cells after oxaliplatin treatment (200x magnification). ${ }^{\star \star} p<0.01$, ${ }^{* \star *} \mathrm{p}<0.001,{ }^{*} * \star \mathrm{p} p 0.0001$. (e) HN1 knockdown deceased clonogenicity of HCC cells under low-dose oxaliplatin treatment. ${ }^{*} \mathrm{p}<0.05$. ( $\left.\mathrm{f}-\mathrm{h}\right)$ The biomarkers of DNA damage ( $\mathrm{\gamma}-\mathrm{H} 2 \mathrm{AX}$ foci) and chromatin relaxation (acetylation of $\mathrm{H} 3$ and $\mathrm{H} 4$ ) in $\mathrm{HN} 1$ knockdown $\mathrm{HCC}$ cells and negative control under timedependent and concentration-dependent treatment of oxaliplatin were analyzed by western blot. (i) Accumulation of DNA damage ( $\mathrm{Y}-\mathrm{H} 2 \mathrm{AX}$ foci) after HN1 knockdown in oxaliplatin-treated HCC cells was analyzed by immunofluorescent staining. ${ }^{*} p<0.05,{ }^{*} p<0.01$. (j) Apoptosis rate of HN1 knockdown and negative control HCC cells was analyzed by flow cytometry. ${ }^{*} p<0.05,{ }^{* *} p<0.01$. 

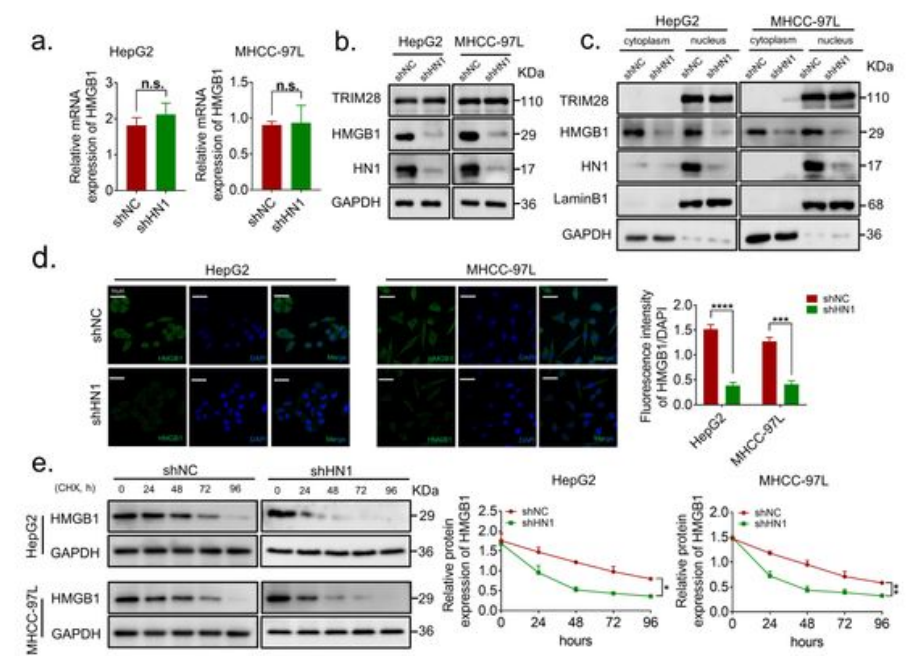

f.
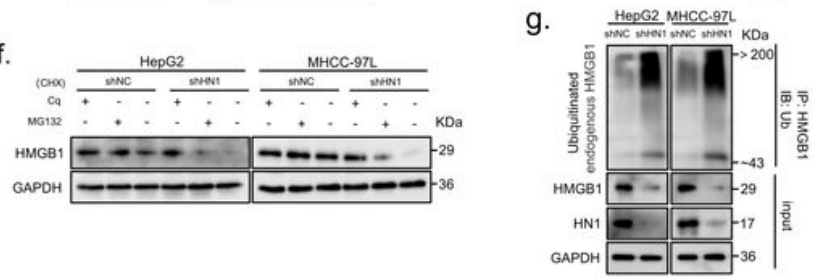
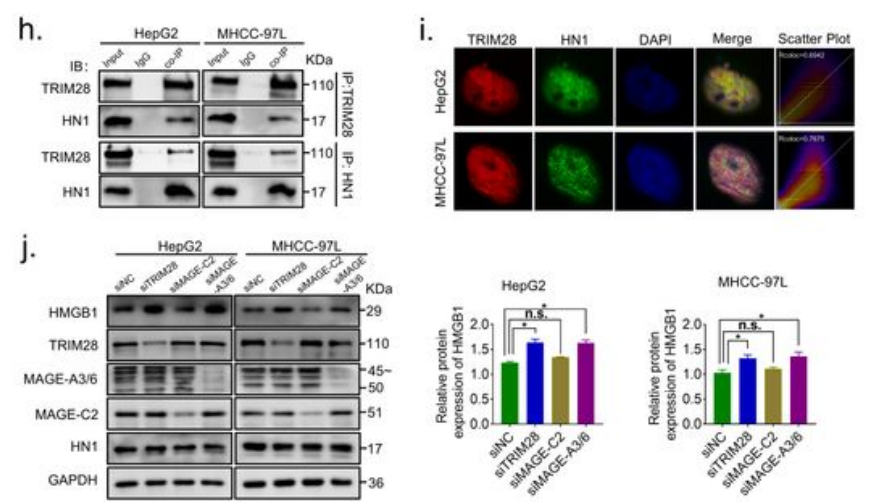

k.

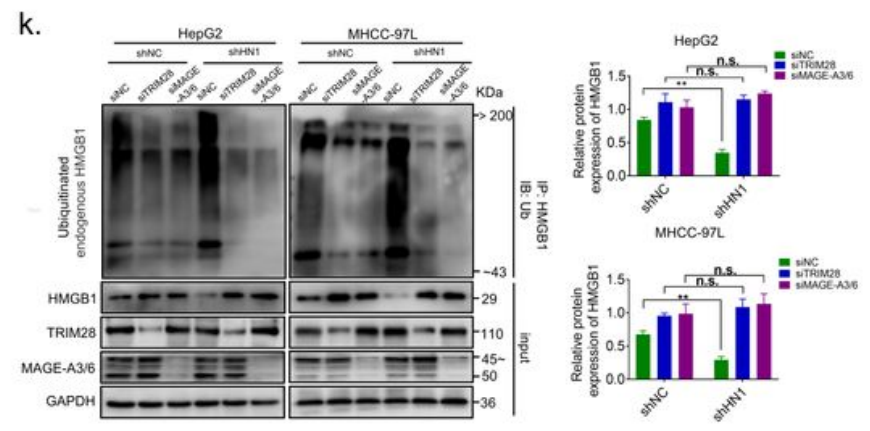

\section{Figure 4}

HN1 inhibited the degradation of HMGB1 through autophagy-lysosome pathway by interacting with TRIM28. (a) The relative mRNA expression of HMGB1 was measured by qRT-PCR in HN1 knockdown and negative control cells. (b-d) The protein level of HMGB1 in HN1 knockdown and negative control HCC

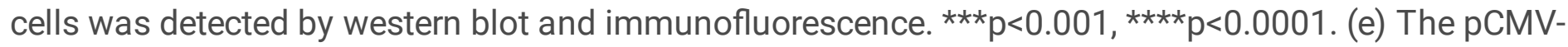
HMGB1 expression plasmid was transfected into shNC and shHN1 cells. After HMGB1 protein was expressed stably for 2 days, cycloheximide $(\mathrm{CHX})(2 \mu \mathrm{g} / \mathrm{mL})$ was added for incubation $24 \mathrm{~h}, 48 \mathrm{~h}, 72 \mathrm{~h}, 96 \mathrm{~h}$ respectively. The degradation rate of HMGB1 was measured by western blot. ${ }^{*} p<0.05,{ }^{*} p<0.01$. (f) The pCMV-HMGB1 expression plasmid was transfected into shNC and shHN1 cells. The protein level of HMGB1 was measured by western blot after chloroquine $(10 \mu \mathrm{M})$ or MG132 $(1 \mu \mathrm{M})$ with $\mathrm{CHX}(2 \mu \mathrm{g} / \mathrm{mL})$ were employed for $24 \mathrm{~h}$ as indicated. (g) Ubiquitinated endogenous HMGB1 was detected by IP assay. (h) The co-IP analysis showed that HN1 interacted with TRIM28. (i) The immunofluorescence co-localization of HN1 and TRIM28 was illustrated in the merged images. Scatter plot indicated a high degree of colocalization. Rcoloc means Pearson's coefficient for image above thresholds. (j) The protein level of HMGB1 in HCC cells with TRIM28 or MAGE-C2 or MAGE-A3/6 knockdown was measured by western blot. ${ }^{*} p<0.05$. (k) HMGB1 protein level and its endogenous ubiquitination level in HN1 knockdown HCC cells with knockdown of TRIM28 or MAGE-A3/6 was measured by western blot. ${ }^{*} p<0.01$. 
a.
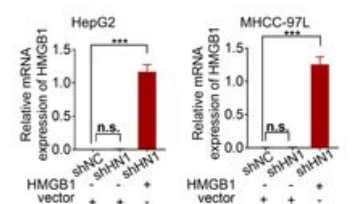

c.

d.
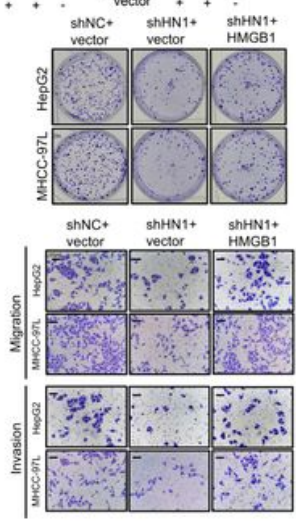

e.

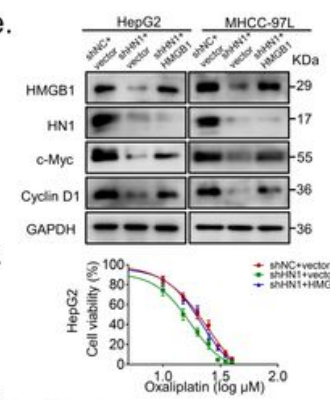

g.

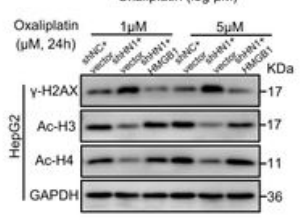

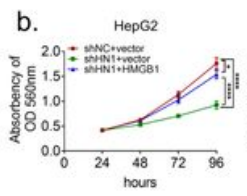
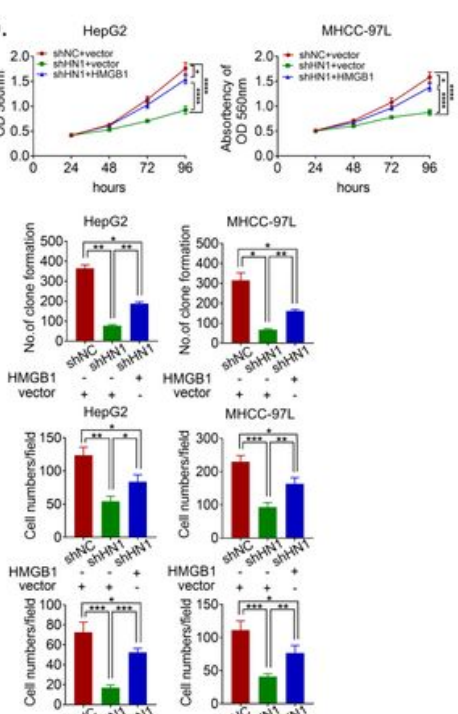

HMGB:
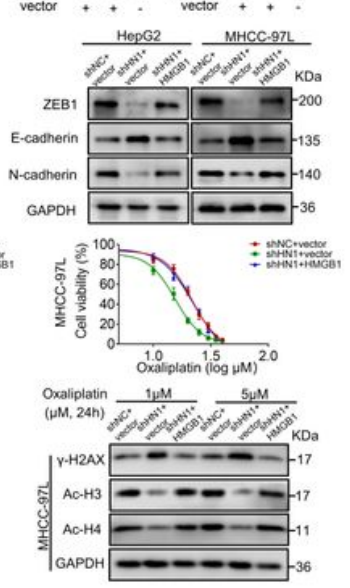

h.
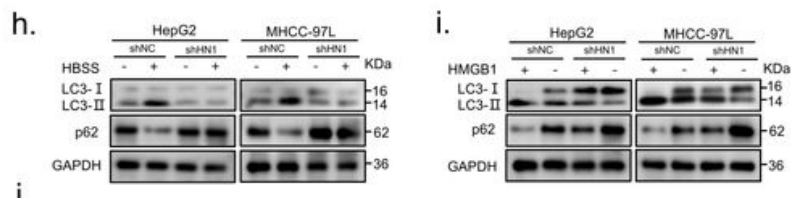

j.
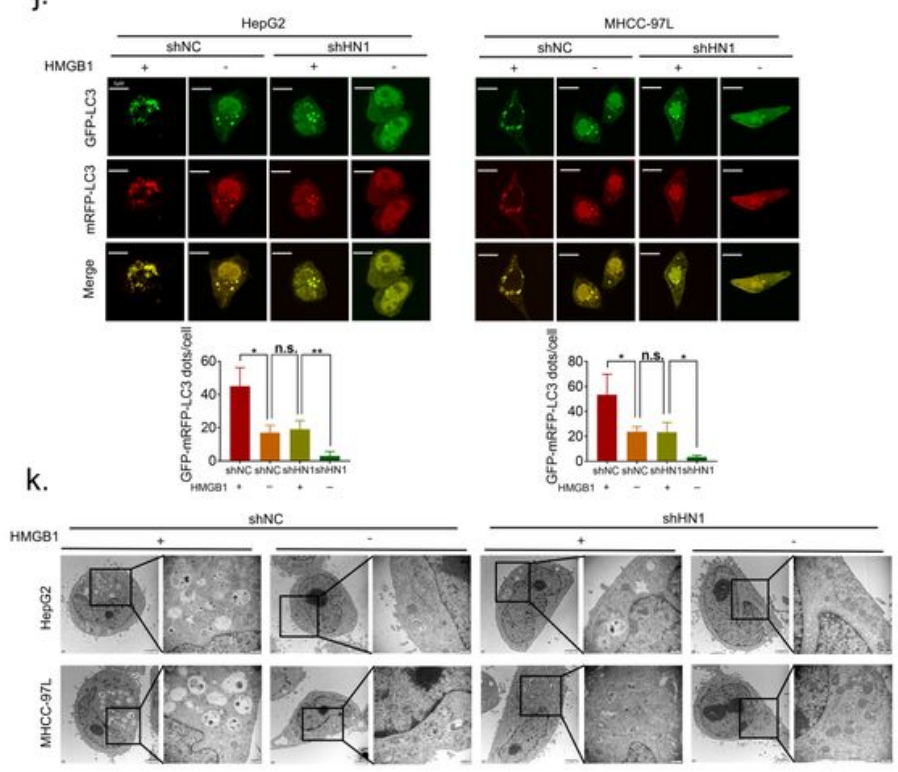

\section{Figure 5}

Overexpression of HMGB1 restored the phenotypes resulted from HN1 knockdown in HCC cells. (a) The relative mRNA expression of HMGB1 was analyzed by qRT-PCR after HMGB1 overexpression. ${ }^{\star * \star} p<0.001$ (b, c) Cell proliferation potency of HN1 knockdown HCC cells with HMGB1 overexpression was measured by MTT assay and clone formation assay. ${ }^{*} p<0.05$, ${ }^{\star \star} p<0.01,{ }^{\star * \star *} p<0.0001$. (d) The potency of migration and invasion of HN1 knockdown HCC cells with HMGB1 overexpression was measured by Transwell assay (400x magnification). ${ }^{\star} p<0.05,{ }^{* \star} p<0.01,{ }^{\star \star *} p<0.001$. (e) The protein levels of HMGB1 and representative biomarkers of cell proliferation and metastasis in HN1 knockdown HCC cells with HMGB1 overexpression were analyzed by western blot. (f) HMGB1 overexpression restored the sensitization of HCC cells to oxaliplatin in HN1 knockdown cells. (g) $\mathrm{y}-\mathrm{H} 2 \mathrm{AX}$ and chromatin relaxation biomarkers were measured by western blot in HN1 knockdown HCC cells with HMGB1 overexpression under treatment of oxaliplatin $(1 \mu \mathrm{M}$ or $5 \mu \mathrm{M}$ for $24 \mathrm{~h})$. (h) LC3-I/II and p62 protein level were tested by western blot in shNC and shHN1 cells starved by HBSS for 3 hours. (i) LC3-I/II and p62 protein level were tested by western blot after PCMV-HMGB1 expression plasmid was transfected into shNC and shHN1 cells and cells were exposed to $0.1 \mu \mathrm{M}$ of bafilomycin A1 for 6 hours before total protein was collected. (j) pCMV-HMGB1 expression plasmid was transfected into shNC and shHN1 cells as indicated and then GFP-mRFP-LC3 adenovirus infected the cells. Bafilomycin A1 $(0.1 \mu \mathrm{M}$ for $6 \mathrm{~h})$ was added before cells were 
fixed. Representative images of LC3 puncta were acquired from confocal microscopy. The GFP-mRFPLC3 puncta meaning the formation of autophagosome was counted and analyzed. ${ }^{*} p<0.05,{ }^{*} p<0.01$. (k) Typical ultrastructural images of shNC or shHN1 cells transfected with pCMV-HMGB1 expression plasmid after treatment of bafilomycin $\mathrm{A} 1(0.1 \mu \mathrm{M}$ for $6 \mathrm{~h})$. More autophagosomes were discovered in shHN1 cells with HMGB1 overexpression compared with single HN1 knockdown cells.

a.

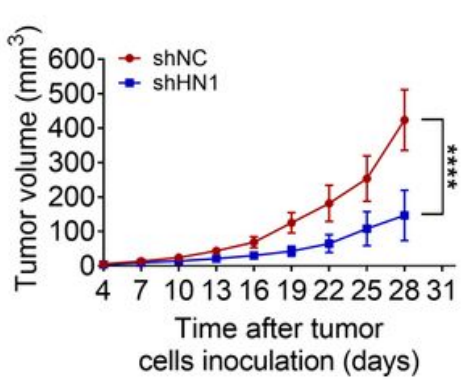

b.

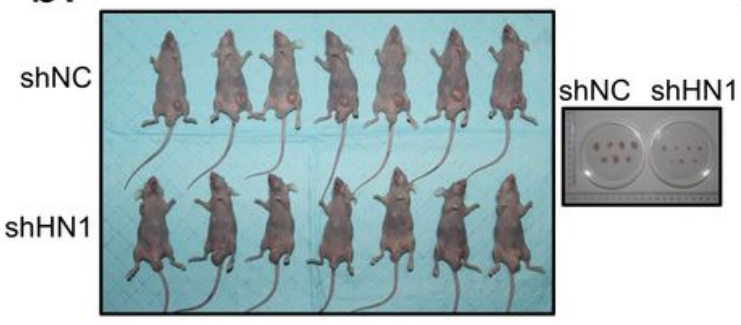

C.

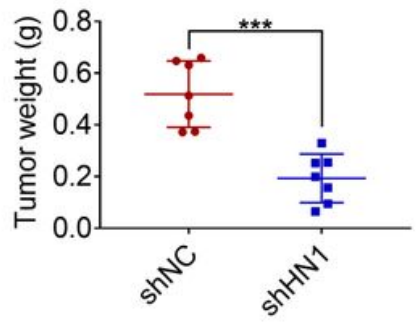

d.
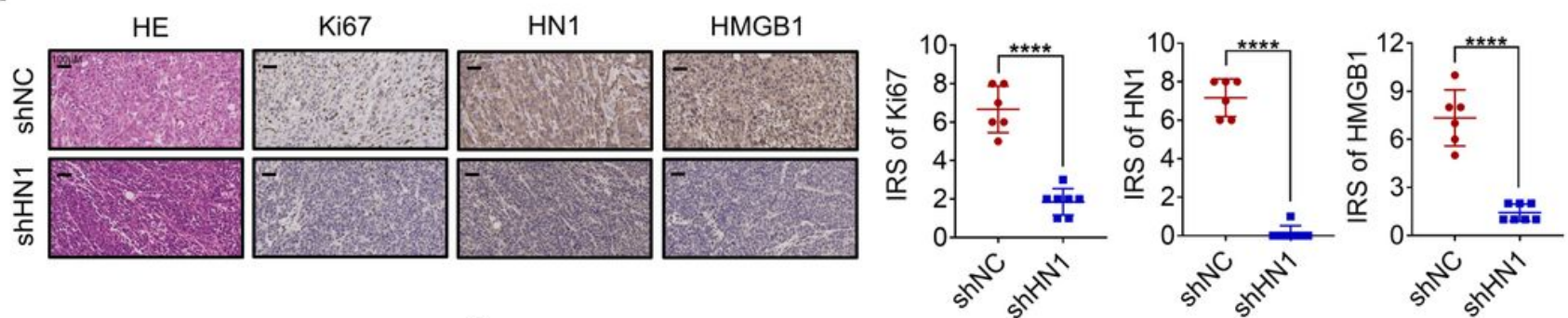

e.

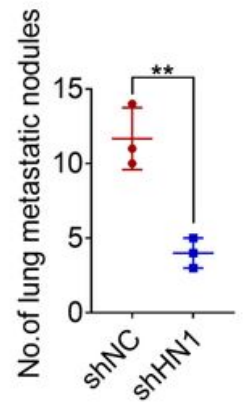

g.

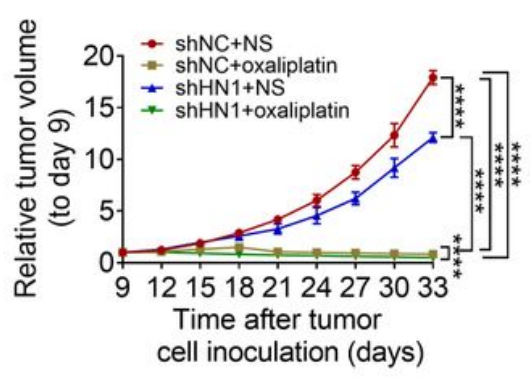

h.

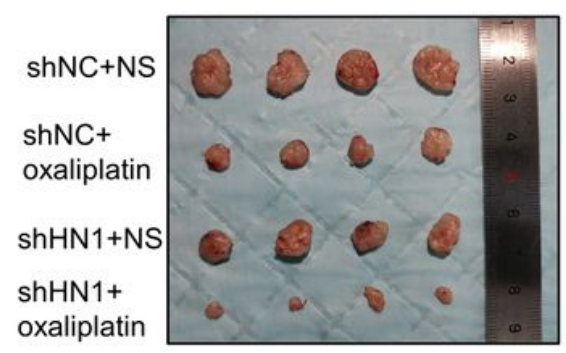

f.

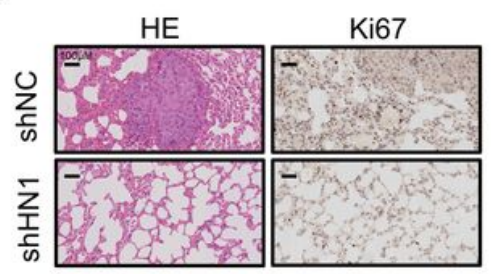

i.

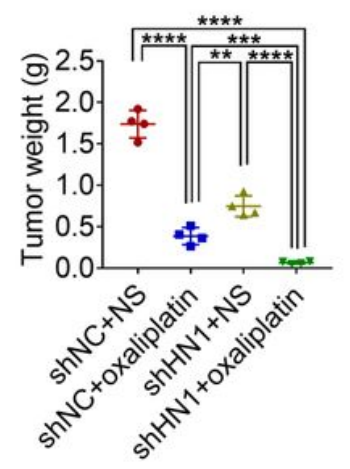

\section{Figure 6}

HN1 knockdown inhibited malignant phenotypes and enhanced the antitumor effect of oxaliplatin in vivo. (a-c) Tumor volume, images of tumor, tumor weight of MHCC-97L cells stably transfected with shNC or 
shHN1 in BALB/c nude mice. ${ }^{* \star} \mathrm{p}<0.001,{ }^{* \star * \star} \mathrm{p}<0.0001$. (d) HN1 and HMGB1 protein level and Ki67 expression in subcutaneous xenografts were detected by immunohistochemistry (200x magnification). $\star \star \star \star x<0.0001$. (e) Representative image and statistical analysis of metastatic nodules of pulmonary metastatic models. ${ }^{*} \mathrm{p}<0.01$. (f) Ki67 expression of metastatic nodules in the lungs was measured by immunohistochemistry (200x magnification). ${ }^{*} \mathrm{p}<0.05$. ( $\left.\mathrm{g}-\mathrm{i}\right)$ Relative tumor volume (to day 9 after inoculation), image of tumor, tumor weight of MHCC-97L cells stably transfected with shNC or shHN1 in BALB/c nude mice treated with oxaliplatin $(10 \mathrm{mg} / \mathrm{kg})$ or NS as control respectively. ${ }^{\star \star} p<0.01,{ }^{\star \star \star} p<0.001$, $\star \star \star \star x<0.0001$.

\section{Supplementary Files}

This is a list of supplementary files associated with this preprint. Click to download.

- Additionalfile1.docx

- Additionalfile2.docx

- Additionalfile3.docx

- Additionalfile4.docx

- Additionalfile5.docx 